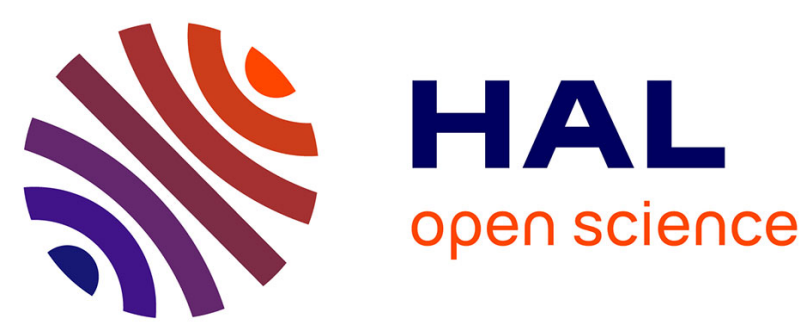

\title{
Modelling of solid particle aggregation dynamics in non-wetting liquid medium
}

Michel Cournil, Frédéric Gruy, Pascal Gardin, Hubert Saint-Raymond

\section{To cite this version:}

Michel Cournil, Frédéric Gruy, Pascal Gardin, Hubert Saint-Raymond. Modelling of solid particle aggregation dynamics in non-wetting liquid medium. Chemical engineering progress, 2006, 45 (7), pp.586-597. 10.1016/j.cep.2006.01.003 . emse-03435378

\section{HAL Id: emse-03435378 https://hal-emse.ccsd.cnrs.fr/emse-03435378}

Submitted on 18 Nov 2021

HAL is a multi-disciplinary open access archive for the deposit and dissemination of scientific research documents, whether they are published or not. The documents may come from teaching and research institutions in France or abroad, or from public or private research centers.
L'archive ouverte pluridisciplinaire HAL, est destinée au dépôt et à la diffusion de documents scientifiques de niveau recherche, publiés ou non, émanant des établissements d'enseignement et de recherche français ou étrangers, des laboratoires publics ou privés. 


\title{
Modelling of solid particle aggregation dynamics in non-wetting liquid medium
}

\author{
Michel COURNIL(*), Frédéric GRUY \\ Ecole des Mines de Saint-Etienne, 158, Cours Fauriel, 42023 Saint-Etienne Cedex 2 (France) \\ Pascal GARDIN, Hubert SAINT-RAYMOND, \\ IRSID (ARCELOR Research), Voie Romaine, BP 30320, 57283 Maizières lès Metz (France)
}

\begin{abstract}
The aim of this work is to re-examine and, if need be, to discuss or modify several of the elements of the classical aggregation dynamics models and to adapt them to the case of aggregation in non-wetting media and particularly alumina inclusions aggregation in a turbulent flow of liquid steel. As proved by several experiments and models, solid particles in contact in non-wetting media are linked by gaseous bridges which may pre-exist prior to the aggregate formation. In this paper, the role of these gaseous cavities in the aggregation process is considered. In particular, a complete calculation of the interaction force between hydrophobic surfaces (Bjerkness- Ruckenstein model) is performed. Main aspects of the aggregation dynamics are envisaged in the particular conditions which result from nonwetting. Hydrodynamic interactions between particles are modified by non-wetting, thus the collision efficiency coefficient and the aggregation kernel. The fragmentation kernel of the aggregates, however, is modified to a larger extent and is equal to zero in most cases. Numerical applications are presented in the reference case (alumina particles in liquid steel). A general procedure of use of this model in other non-wetting situations is given too.

(*) corresponding author: cournil@emse.fr

Phone: ++33 (0) 477420150
\end{abstract}




\section{INTRODUCTION}

Aggregation of hydrophilic particles in stirred liquid media can be considered as a relatively well understood process in spite of the variety and complexity of its aspects. Good models exist in particular for representing the physicochemical interactions between aggregates and for predicting the collision rates and their efficiency [1-9]. The procedure proposed by Kusters et al [8] has revealed so far particularly efficient to take into account the porous character of the aggregates in a comprehensive dynamical model.

Aggregation of solid particles in non wetting media is less known, at least on certain aspects. A large number of experimental works indeed have definitely proved the existence of strong long range (20-200 nm) attractive forces between hydrophobic surfaces in water [10-13]. Their most likely explanation focuses upon the bridging of nanobubbles which pre-exist on the hydrophobic surface. The existence of these bubbles has been first deduced from force measurements and then confirmed by direct observations [14-18]. Concerning the aggregation process itself, these bubbles play a major role, in bridging the particles which have entered in contact. Hydrodynamic aspects are also relatively well known: drag force on hydrophobic particle, repulsive hydrodynamic force between hydrophobic particles in motion.

In recent works, we investigated two experimental situations of solid aggregation in nonwetting conditions. The first one concerns "clean" steel production [19]. Steel-making processes include a de-oxidation step in which a reducing agent, Aluminium, for instance, is added to the liquid steel. Consequence is the formation in the bath of metal oxide particles, typically, 3 to $10 \mu \mathrm{m}$ alumina inclusions. Observations show that these inclusions tend to gather and to form ramified clusters of 50 to $300 \mu \mathrm{m}$ which keep a strong cohesion in spite of 
the highly turbulent conditions created by the melt flow in certain parts of the reactor. These clusters are responsible for defects which may seriously alter the steel mechanical properties. The conditions of formation and the characteristics of these aggregates have given rise to several works for many years [20-22]. Assumption of gas bridges between particles was put forward in this case too to explain the high cohesion and size of the observed agglomerates. We re-visited recently this problem [19]. Because of the lack for results due to the difficult experimental conditions (temperature $2000 \mathrm{~K}$ ), we developed the analogy with aggregation of hydrophobic silica particles in water-ethanol solution [23]. In particular, we clearly proved that the unusual optical properties of the aggregates could be explained by the invasion of their structure by gas pockets.

For practical reasons of product quality, process control, equipment sizing and design, comprehensive models of aggregation dynamics in non-wetting media are becoming necessary. To our knowledge, however, no quantitative predictive model - we mean comparable to Kusters approach [8] - is presently available to analyse and interpret aggregation in non-wetting media.

From a general point of view, aggregation models should take into account the following aspects [7-9]: i) nature and intensity of physicochemical interactions between separate solid particles at rest; ii) collision frequency; iii) collision efficiency; iv) link creation between particles; v) fragmentation; vi) aggregate morphology.

The aim of this work is to re-examine and, if need be, to discuss or modify several of the elements of the classical aggregation models and to adapt them to the case of aggregation in non-wetting media and particularly alumina inclusions aggregation in a turbulent flow of liquid steel. 
A simplified version of this model has been applied to the case of hydrophobic silica aggregation [23] in aqueous media; however its complete development has never been published extensively yet.

\section{GEOMETRICAL CONFIGURATION AND INTERACTION FORCES}

\section{Three-phase systems in case of non wetting}

For the reference system, alumina (S), liquid steel (L) and gas (G), respective interfacial tensions at the operating temperature of $2000 \mathrm{~K}$ are: $\gamma_{\mathrm{SG}}=0.65 \mathrm{~J} . \mathrm{m}^{-2}, \gamma_{\mathrm{LG}}=1.70 \mathrm{~J} . \mathrm{m}^{-2}, \gamma_{\mathrm{SL}}=$ $1.96 \mathrm{~J} \cdot \mathrm{m}^{-2}$. Equilibrium of the contact line between the three phases gas-liquid-solid (when it exists) imposes the Young relation:

$$
\gamma_{\mathrm{SG}}-\gamma_{\mathrm{SL}}=\gamma_{\mathrm{LG}} \cos \theta
$$

In the present case, $\theta=140^{\circ}\left(2.44\right.$ radians). Contact angle is greater than $90^{\circ}$, as expected in case of non-wetting.

The presence of bubbles at the surface of hydrophobic surfaces in water has been first deduced from force measurements and then confirmed by direct observations [17-18]. Two different configurations of gas - liquid - solid systems are shown in Figure 1 because of their possible role in aggregation in non-wetting media. The existence and evolution possibility of each configuration can be determined from thermodynamic considerations using the Gibbs enthalpy of formation $\Delta G_{\mathrm{n}}$ of the gas-liquid-solid system [17, 24-25].

$$
\Delta G_{n}=n \boldsymbol{R} T \ln \frac{P_{e x t}}{P_{s a t}}+\gamma_{L G}\left(\Delta S_{L G}+\Delta S_{S G} \cos \theta\right)
$$

$n$ is the mole number in the gas phase, $T$, the temperature, $R$, the gas constant, $P_{\text {ext }}$, the external pressure, $P_{\text {sat, }}$, the saturation pressure of liquid at $2000 \mathrm{~K} . \Delta S_{L G}$ and $\Delta S_{S G}$ are the respective changes in liquid-gas and solid-gas interfacial areas. 
* spherical bubble on a solid support (Figure 1a)

Concerning convex bubbles, as $\Delta G_{n}$ and $\mathrm{d}\left(\Delta G_{n}\right) / \mathrm{d} n$ are positive, neither nucleation nor development of such bubbles are spontaneous. In the actual conditions of steel melts which are generally stirred by argon flow injection, free bubbles may pre-exist, however, and be captured by the solid surfaces.

* gas cavity in a pore

The possibility of formation of a gas cavity in a pore or a crevice of the solid surface is considered now. This geometrical configuration has not been envisaged so far in the literature. We first assume that the crevice has flat walls, which correspond to a cylindrical concave liquid-gas interface (Figure 1b)

The half-angle of the crevice is denoted $\alpha(\alpha<\theta-\pi / 2)$, the "dry" length, $h_{d}$, the transversal width, $L . V_{\mathrm{G}}$ and $P_{\mathrm{G}}$ are the respective gas phase volume and pressure. After expressing the different geometric parameters of this configuration and putting them into expression (2) of $\Delta G_{n}$, we obtain:

$\Delta G_{n}=P_{G} V_{G} \ln \frac{P_{\text {ext }}}{P_{\text {sat }}}+h_{d} L\left[-\frac{\sin \alpha}{\cos (\theta-\alpha)}(2 \theta-2 \alpha-\pi)+2 \cos \theta\right] \gamma_{L G}$

In the system considered here, the solid phase generally consists of small inclusions of typical size $5 \mu \mathrm{m}$ It is likely that vapour pressure in the cavity does not fall below the saturation value $P_{\text {sat }}$; thus, in what follows, in agreement with $[12,25]$ we will assume that $P_{\mathrm{G}}=P_{\text {sat. }}$.

We come back now to the thermodynamic meaning of $\Delta G_{n}$. From basic mathematical analysis of Eqn (3), it follows that: $\Delta G_{n}$ is zero for $h_{d}=0$, then decreases at increasing $h_{d}$, thus takes negative values while $h_{d}$ is lower than threshold value $h_{\mathrm{c}}$ which is much larger than the inclusion size (for $\alpha=5^{\circ}$ and the reference system $h_{\mathrm{c}}=0.11 \mathrm{~m}$ !). According to the previous thermodynamic criteria, it follows that gas cavities can spontaneous nucleate and grow in 
most of the pores, holes or crevices of the inclusion surface and probably fill them to reach the external surface. This seems to be a general result. For instance it is also verified with conical pores. In both cases, the same geometrical constraint: $\alpha<\theta-\pi / 2$ (that is to say: $\alpha<$ $50^{\circ}$ for alumina in liquid steel) should be verified.

Thus we propose to consider that the surface of a solid particle immersed in a non-wetting liquid is partially covered with a gas layer which consists of the upper interface of different cavities filled with gas (Figure 2).

This situation is quite consistent with the most recent models and experimental observations $[14,18,25-26]$

\section{Interactions between separate solid particles at rest in non-wetting medium}

From a general point of view, motionless particles in interaction in a fluid medium are submitted to the respective forces of London-Van der Waals attraction and electrochemical double layer repulsion [27, 28].

In the present case of non-aqueous medium, Van der Waals forces are still active, whereas electrochemical forces are irrelevant. We will also show that presence of gas bubbles or cavities at the particle surfaces results in attractive forces which will be estimated below.

\section{- Van der Waals force}

This force, $F_{\mathrm{w}}$, has its origin in interactions between instantaneous induced dipoles [28]. For two spheres of radii $R$ separated by a distance $h$; it is given by:

$$
F_{\mathrm{W}}=\frac{A}{6 R} P\left(2+\frac{h}{R}\right)
$$

Function $P$ is defined by: 


$$
P(s)=-\frac{4 s}{\left(s^{2}-4\right)^{2}}-\frac{4}{s^{3}}+\frac{2 s}{s^{2}-4}-\frac{2}{s}
$$

$A$, the Hamaker constant can be calculated according two methods [29, 30]. To our knowledge, its determination for alumina particles in molten steel has never been done before. The Lifshitz method, which we preferred in our calculations, only requires the knowledge of the relative permittivity of the different media. For sake of space, we only give the result of the calculation, i.e.:

$$
A=1.0 \times 10^{-19} \mathrm{~J}
$$

This is a common value for Hamaker constant of a solid compound in a liquid phase.

\section{- interaction forces in case of non-wetting}

As said before, strong attractive forces between hydrophobic particles in water are generally associated with the presence of micro- or nanobubbles at their surface. This explanation, however, often suggests prior contact or nearly-contact between particles to allow gaseous bridging whereas several experiments seem to prove that the interaction exists before. A few theoretical works propose different possible interpretations for the "hydrophobic" force:

i) assumption of a force from electrostatic origin seems to have been definitively abandoned [31] because of insensitivity to the Debye length of the liquid solution and anyway is not relevant in case of non-aqueous media.

ii) other models take into account the solvent structure in the vicinity of the interface and particularly the solvation effect; in these conditions, it appears that hydrophobicity can result in a decrease in the solvent density and an increase in the attractive forces $[32,33]$. The range of this effect however is not beyond $4 \mathrm{~nm}$, thus is not sufficient to explain interactions still effective up to several tens of nanometers. 
iii) mechanical origin: $[25,26,34]$ have calculated interactions between surfaces covered with gaseous gaps, however separated by a liquid layer. They do not agree on the origin of the interaction: elastic propagation for Yaminski and Ninham [25], hydrodynamic interactions between pulsating bubbles for [26, 34]. These two models predict a long range attractive force and remain realistic when the liquid phase consists of molten steel. The latter gives a better interpretation of the temperature dependence of the hydrophobic force, thus will be developed quantitatively in the follow-up:

\section{- calculation of the interaction force (Bjerkness force)}

\section{calculation principle}

We refer here to the approach of $[26,34]$ which attributes a hydrodynamic origin to the interaction force between hydrophobic surfaces in water. Starting point of this model is the Bjerkness force which is known to appear between two oscillating bubbles (A and B) in a liquid and is equal to:

$$
f_{B}=\frac{2 \pi r_{b A}^{2} r_{b B}^{2} \delta_{A} \delta_{B} \rho \omega^{2} \cos \varphi}{r^{2}}
$$

In this relation, $\rho$ is the liquid phase density, $r_{b A}$ and $r_{b B}$, the respective bubble radii, $\delta_{A}$ and $\delta_{B}$, the respective bubble oscillation amplitudes; $\omega$ the pulsation and $r$ the separation distance between bubbles and $\varphi$ the phase difference.

Assuming identical bubbles and zero phase difference, Equation (6) becomes:

$$
f_{B}=\frac{2 \pi r_{b}^{4} \delta^{2} \rho \omega^{2}}{r^{2}}
$$

Considering that each particle surface is covered with identical pulsating gas bubbles or cavities, next steps of total force calculation are:

i. expression of unknown parameters $r_{b}, \omega$ and $\delta$ 
ii. integration of Equation [6] over all the bubble pairs

We do not adopt strictly the approach of Ruckenstein's [34] work for three reasons:

- in his work, $r_{b}, \omega$ and $\delta$ are essentially derived from dimensionless analysis, whereas in the present paper other methods of determination are proposed (see subsection: parameter estimation);

- viscosity effect on interaction between particles can be stated more precisely;

- integral calculation in [34] seems us doubtful and no quantitative values of the resulting forces are obtained.

\section{parameter estimation}

Bubble radius $r_{b}$ is imposed by the porosity or similarly roughness scales. Both should vary in the range: $\left[r_{b 1}=10^{-8} \mathrm{~m} ; r_{b 2}=10^{-7} \mathrm{~m}\right]$. Bubble pulsations take their origin in the local thermal fluctuations; their relative amplitude $\beta=\frac{\delta}{r_{b}}$ should range between $10^{-2}$ and $10^{-1}$.

This variation range is roughly estimated. It takes into account the bubble inertia. Larger values of oscillation amplitude are possible however not necessary for bubbles to establish bridges between the two surfaces. From thermodynamic reasoning, it is possible to prove that these sort of bridges spontaneously develop around the contact points between particles. In this section we only insist on the fact that small oscillations may bring about noticeable Bjerknes forces.

Lastly, $\omega$ is the pulsation value corresponding to the proper oscillation frequency of the bubble, i.e.:

$$
\omega=\sqrt{\frac{\gamma_{L G}}{\rho r_{b}^{3}}}
$$


For the system investigated here, $\omega$ varies between $\omega^{\prime}=4.5 \times 10^{8} \mathrm{~s}^{-1}$ and $\omega^{\prime \prime}=1.4 \times 10^{10} \mathrm{~s}^{-1}$ (values respectively taken for $r_{b 2}$ and $r_{b 1}$ ).

viscosity role

Viscosity is well known for its damping effect on the wave amplitudes which rapidly decreases with distance $r$ from the pulsating surface according to an exponential law in $\mathrm{e}^{-r / d}$ [35]. Depth penetration $d$ is given by:

$$
d=\sqrt{\frac{2 \mu}{\rho \omega}}
$$

Here, for a dynamic viscosity of $4.9 \times 10^{-3} \mathrm{~kg} \cdot \mathrm{m}^{-1} \cdot \mathrm{s}^{-1}, d$ varies between $d_{1}=1.0 \times 10^{-8} \mathrm{~m}$ and $d_{2}=5.7 \times 10^{-8} \mathrm{~m}$

From (7), (8) and (9) follows:

$$
f_{B}=\frac{\pi r_{b}^{3} \gamma_{L G} \beta^{2}}{r^{2}} \exp \left(-\frac{r}{d}\right)
$$

Factor 2 has been omitted because only half part of each bubble is affected by the interaction.

integration over two hemispherical surfaces

We consider now the gaseous cavities located in the pores of two identical spherical particles distant from separation $h$ (Figure 3); we denote by $\varepsilon_{p}$ the surface coverage ratio by these pores and by $\varepsilon$ ' the ratio of these pores actually filled with gas ("active" pores). As the formation of gas bubbles in the pores is always thermodynamically possible, $\varepsilon^{\prime}$ is close to 1 . The number of gas cavities per unit surface area is then:

$$
n_{b}=\frac{\varepsilon_{p} \varepsilon^{\prime}}{\pi r_{b}^{2}}
$$

Integration of Equation (11) over the active pores gives the interaction force between the spherical particles: 


$$
F_{B}=\frac{1}{2} \iint \frac{2}{\pi r_{12}^{2}} \varepsilon_{P}^{2} \varepsilon^{2} \delta^{2} \rho \omega^{2} \cos \theta_{1} \cos \theta_{2} \frac{\overrightarrow{r_{12} u}}{r_{12}} e^{-\frac{r_{12}}{d}} \mathrm{~d} S_{1} \mathrm{~d} S_{2}
$$

Subscripts 1 and 2 refer to spheres 1 and 2. Integration concerns surfaces facing each other. $\overrightarrow{r_{12}}$ is the vector joining a point of surface 1 and a point of surface $2 . \vec{u}$ is the unit vector of the straight line which connects the centres of the two spheres. $\theta_{1}$ denotes the angle between the normal to the sphere at point $M_{1}$ and vector $\overrightarrow{r_{12}}$ (resp. $\theta_{2}$ and $M_{2}$ ).

After adimensioning all lengths (including $d$ ) by division by radius $R$, we obtain:

$$
F_{B}=\frac{1}{\pi} \varepsilon_{p}{ }^{2} \varepsilon^{\prime 2} \gamma_{L G} \beta^{2} \frac{R^{2}}{r_{b}} I
$$

with $I=\iint \cos \theta_{1} \cos \theta_{2} \mathrm{e}^{-\frac{r_{12}}{d}} \frac{\vec{u} \cdot \overrightarrow{r_{12}}}{r_{12}{ }^{3}} d S_{1} d S_{2}$

$I$ is a dimensionless integral.

This interaction force can be now compared to the van der Waals interaction $(4,5)$ :

$$
r_{B / W}=\frac{F_{B}}{F_{W}}=\xi \frac{I(h, d)}{P(2+h)}
$$

with:

$$
\xi=\frac{6}{\pi} \varepsilon_{p}{ }^{2} \varepsilon^{\prime 2} \beta^{2} \frac{\gamma_{L G} R^{3}}{A r_{b}}
$$

\section{numerical application :}

We propose now to compare $F_{\mathrm{B}}$ and $F_{\mathrm{W}}$, taking the following set of numerical values which have been justified above in this paper.

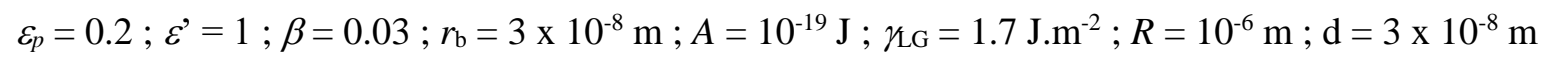


Whatever $d$ values, a maximum of $F_{\mathrm{B}} / F_{\mathrm{W}}$ is observed for $h=2 d$. Other numerical works, not presented here, prove that the maximum value of $F_{\mathrm{B}} / F_{\mathrm{W}}$ is little sensitive to particle radius $R$. Moreover, maximum value of $F_{\mathrm{B}} / F_{\mathrm{W}}$ is an increasing function of $d$ (Table 1). From these results, it is clear that if $d<0.01 \mu \mathrm{m}$, Bjerkness force is negligible when compared to van der Waals force. On the contrary, if $d>0.1 \mu \mathrm{m}$, van der Waals force becomes negligible when compared to Bjerkness force. Our reference experimental case ( $d$ from $0.01 \mu \mathrm{m}$ to $0.06 \mu \mathrm{m}$ ) is in-between, thus: $0.1<F_{\mathrm{B}} / F_{\mathrm{W}}<40$.

This means that van der Waals and Bjerkness forces are of similar order of magnitude in the reference case. We will come back further to the actual influence of such forces on aggregation dynamics.

\section{Forces between particles linked by a gas bridge in a non-wetting medium}

Configuration in which the two particle surfaces are linked by a toroidal gas bridge is considered now (Figure 4). Problem of determination of forces due to the presence of such material bridge between solid particles has been studied by many authors. Most of the works were devoted to the determination of the capillary binding forces due to liquid bridges [3638]. Case of gaseous bridges is quite similar [10-12].

In all these works, force $F_{G}$ between two particles is broken down into two contributions: the capillary force $F_{1}$ due to the surface tension of the bridge and the pressure force $F_{2}$ due to the pressure deficiency in the bridge. After some controversies on the most exact way to calculate these forces, particularly in the framework of the toroidal approximation, it seems now that the predictions of the "gorge method" [38] are considered as the best when compared to experimental results. The "gorge method" consists in calculating the previous two forces at the neck of the bridge. For geometry of Figure 4 relative to two plane surfaces connected by a gaseous bridge, this gives: 


$$
\begin{aligned}
& F_{1}=2 \pi r_{1} \gamma_{\mathrm{LG}} \\
& F_{2}=\pi r_{1}{ }^{2} \gamma_{\mathrm{LG}}\left(\frac{1}{r_{2}}-\frac{1}{r_{1}}\right)
\end{aligned}
$$

thus: $\quad F_{G}=\pi \gamma_{\mathrm{LG}} r_{1}\left(\frac{r_{2}+r_{1}}{r_{2}}\right)$

It is now possible to compare $F_{\mathrm{G}}$ to van der Waals force $F_{\mathrm{w}}$, (4) for alumina in liquid steel.

Taking $h=-2 r_{2} \cos \theta$ and $r_{1} \approx r_{2}$, one obtains : $\frac{F_{W}}{F_{G}}=\frac{1.4 \times 10^{-10}}{r_{1}^{2}}$ where $r_{1}$ is expressed in micrometers. This confirms, if need be, that van der Waals forces are negligible once the gas bridge is established; thus the cohesion force of the doublet of particles is considerably higher than in the case of aggregation in wetting conditions. This point will be examined again in the next section.

\section{Aggregation mechanism of solid particles in non-wetting medium}

From Eqn (2), the Gibbs enthalpy of formation of the gas bridge is:

$$
\Delta G_{n}=\pi r_{1}^{2} h P_{G} \log \frac{P_{\text {ext }}}{P_{\text {sat }}}+2 \pi r_{1} \gamma_{L G}\left(h+r_{1} \cos \theta\right)
$$

$\Delta G_{\mathrm{n}}$ is a function of the two independent parameters $r_{1}$ and $h$.

Whatever the value of $P_{\mathrm{G}}, \Delta G_{n}$ is an increasing function of $h$. For zero $h, \Delta G_{n}$ is negative and decreases when $r_{1}$ increases ; for non zero $h, \Delta G_{n}$ first increases and takes positive values when $r_{1}$ increases up to a value $r_{c}^{\prime}$ which is practically equal to : $-h /(2 \cos \theta)$, then $\Delta G_{n}$ decreases and becomes negative beyond a value $r{ }^{\prime}$ of about $-h / \cos \theta$.

From these properties it appears that a gas bridge between two plane surfaces can spontaneous appear only if they are in contact, otherwise, an activation is necessary. Once a gas bridge is 
formed, it can spontaneously evolve in the following way (Figure 4): lateral growth if $r_{1}$ is larger than $r_{c}^{\prime}$, and shrinkage according to $h$ till the surfaces are in contact.

From the previous discussions, it has been possible to determine the main static characteristics of aggregation of solid particles in a non-wetting medium and thus to clarify the conditions of calculation of the aggregation and fragmentation kernels. The main points to highlight are:

i) spontaneous formation of gaseous cavities in the pores which may widely modify the solidliquid interface, conferring it, at least to some extent, the characteristics of a gas-liquid interface;

ii) interaction between particles covered with these gas cavities: a new model of calculation of the resulting static hydrodynamic force is proposed, however, in the investigated system (alumina inclusion in liquid steel), this force is probably of the same order of magnitude as the van der Waals force;

iii) spontaneous bridging between two particles when contact occurs at the level of a gas cavity;

iv) spontaneous lateral development of this bridge over the particle surface;

v) existence of strong forces of cohesion due to this gas bridge.

\section{DYNAMICAL ASPECTS OF AGGREGATION IN NON-WETTING MEDIA}

In this section, we will successively examine the effect of non-wetting and connected phenomena on the aggregation and fragmentation kernels.

\section{Aggregation kernels}

We consider the typical case of $5 \mu \mathrm{m}$ solid grains in a turbulent medium with a $50 \mu \mathrm{m}$ Kolmogorov scale. In these conditions, particle motion is imposed by liquid motion and not by Brownian agitation. Moreover, at the eddy scale, the solid particles can be considered as 
submitted to a local laminar flow characterised by a velocity gradient $\dot{\gamma}$. Two particles located on neighbouring streamlines follow parallel trajectories, however with different velocities which make possible their collision.

If we consider a suspension consisting of several classes of particles or aggregates (mean radius $R_{\mathrm{i}}$ and density by number $n_{\mathrm{i}}$ ), the collision frequency of particle $\mathrm{i}$ with particle $\mathrm{j}$ can be calculated as follows [1]:

$$
J_{i j}^{0}=\frac{4}{3} \dot{\gamma}\left(R_{i}+R_{j}\right)^{3} n_{j}
$$

In a turbulent flow, $\dot{\gamma}$ is proportional to $\left(\frac{\varepsilon}{v}\right)^{1 / 2}$, where $\varepsilon$ is the turbulent energy dissipation rate per unit mass and $v$ the kinematic viscosity.

Comprehensive aggregation models should take into account the modifications brought to the previous collision frequency by the interaction forces between particles. Some of these forces also exist in motionless system, as we saw before; others, however, appear only with motion (fluid drainage). To take into account these effects, the modified collision frequency is written in the form:

$$
J_{i j}=\alpha_{i j} J_{i j}^{0}
$$

where $\alpha_{\mathrm{ij}}$ is the collision efficiency coefficient.

The main objective of this section is to calculate $\alpha_{\mathrm{ij}}$. This has been already done in the case of wetting liquid, when the conventional no-slip condition is fulfilled $[5,6,9]$.

In case of non-wetting, the presence of a great number of gas bubbles or cavities at the interface may result in noticeable slippage of the liquid. In the case of a sphere immersed in a flow, slippage is characterized by following boundary conditions, at the sphere surface: 


$$
V_{\theta}=b \frac{\tau_{r \theta}}{\mu}
$$

$V_{\theta}$ is the tangential velocity and $\frac{\tau_{r \theta}}{\mu}$ is the projection on the tangential plane of the velocity gradient tensor; $b$ is the so-called slip-length. $b$ is zero in case of no-slip (wetting conditions). Symmetrical extreme case $(b \rightarrow \infty)$ would correspond to gas-liquid interface.

Slippage effects have been already considered in situations of a non-wetting liquid on a solid surface [39-41]. In particular, Vinogradova [41] calculated the hydrodynamic resistance force between two hydrophobic surfaces and showed that in some cases, the approach velocity of particle can drop to zero and aggregation does not occur. However, to our knowledge, collision efficiency $\alpha_{\mathrm{ij}}$ between two particles in non-wetting medium has not been calculated yet. Solving this problem would be particularly useful because aggregation dynamics could be formulated in the same terms both in hydrophobic and hydrophilic case.

$\alpha_{\mathrm{ij}}$ value is determined in several steps:

i) single spherical particle in a flow field [41, 42]

In case of wetting, the force exerted by a fluid of velocity $V_{\infty}$ on a spherical particle at rest of radius $R$ is:

$$
\begin{aligned}
& |\vec{F}|=6 \pi \mu R V_{\infty} \\
& f_{1}=6 \pi \mu R \text { is the friction coefficient. }
\end{aligned}
$$

In case of non wetting:

$$
|\vec{F}|=6 \pi \mu R V_{\infty} \frac{R+2 b}{R+3 b}
$$

thus the friction coefficient $f_{1}^{h}$ : 


$$
f_{1}^{h}=6 \pi \mu R \frac{R+2 b}{R+3 b}
$$

ii) particle doublet in a flow field: case of wetting

Fluid drainage between two particles results in the hydrodynamic resistance force:

$$
F_{s s}=f_{2} v
$$

To incorporate hydrodynamical and physicochemical interactions into the flow-rate calculation in the case of Brownian aggregation, Spielman [4] modified as follows the Smoluchovski expression of aggregation rate in the case of two identical spheres of radius $R$ :

$$
J_{b}=4 \pi r^{2} G(r)\left(\mathrm{D}_{b} \frac{\partial n}{\partial r}+\frac{2 n}{6 \pi R \mu} \frac{\mathrm{d} V}{\mathrm{~d} r}\right)
$$

where $D_{\mathrm{b}}$ is the Brownian diffusion coefficient, $V$ is the interaction potential (from physicochemical origin) and $G(r)$ a correction factor which links friction factor $f_{1}$ of a single particle and factor $f_{2}$ of a doublet:

$$
f_{2}=\frac{f_{1}}{G(r)}=\frac{6 \pi R \mu}{G(r)}
$$

This correction factor expresses the hindrance to collision due to the presence of a liquid layer of thickness $h$ between two particles (hydrodynamic interaction). According to Derjaguin and Müller [43], the easiest way to express $G(h)$ is:

$$
G(h)=\frac{2 h}{R+2 h}
$$

Remark: when $h$ tends to zero $G(h)$ behaves as :

$$
G^{0}(h)=\frac{2 h}{R}
$$

thus $G(h)$ can be written as: 


$$
G(h)=\frac{G^{0}(h)}{1+G^{0}(h)}
$$

In [44] two of us proposed to extend the approach of Spielman to the situation of turbulent aggregation, by only replacing $D_{b}$ for the turbulent diffusivity $D_{t}$.

In the case of London-van der Waals attractive forces, an elementary calculation [44] starting from Eqn (27) leads to the expression of the collision efficiency between two particles:

$$
\alpha_{12}=\left[24 \int_{2}^{\infty} \frac{e^{18 \pi C_{A} \int_{s}^{1} \frac{1}{s^{2}} \frac{\mathrm{d}^{\prime}(s)}{\mathrm{d} s} d s}}{r^{r^{4} G\left(r^{\prime}\right)}} d r^{\prime}\right]^{-1}
$$

where $V^{\prime}$ is the dimensionless interaction potential $V^{\prime}=6 \frac{V}{A}$ and $r^{\prime}=\frac{r}{R}$.

$C_{\mathrm{A}}$ is a dimensionless number which compares physical and hydrodynamic forces:

$$
C_{A}=\frac{A}{36 \pi \mu R^{3} \dot{\gamma}}
$$

The previous development is valid for no-slip conditions at the solid-liquid interface. We will now examine the modifications induced by non-wetting and slippage.

iii) particle doublet in a flow field: case of non-wetting

To introduce modifications due to non-wetting, coefficient $f_{2}^{h}$ expression in the new flow conditions is needed. This calculation can be found in [40].

$$
f_{2}^{h}=\frac{1}{2} \pi R^{2} \frac{\mu}{b}\left[\frac{h+6 b}{6 b} \ln \left(\frac{h+6 b}{h}\right)-1\right]
$$

In the same way as we did in (32), we can define a correction coefficient $G_{h}$ adapted to the case of interaction between two particles in a non-wetting medium:

$$
G_{h}(h)=\frac{f_{1}^{h}}{f_{2}^{h}}
$$


In fact, as expression (34) of $f_{2}^{h}$ is strictly valid for small $\frac{h}{R}$, putting it in (35) will give us $G_{h}^{0}(h)$; at larger interparticular separations, $G_{h}(h)$ is obtained from (31).

Moreover, a slight modification to previous equations is still necessary. We are reasoning here within the context of Spielman theory (two spheres in motion; $G^{0}(h)=\frac{2 h}{R}$ ) whereas Vinogradova $[40,41]$ results refer to only a mobile $\left(G^{0}(h)=\frac{4 h}{R}\right)$. To conciliate the two approaches we have to adopt in the sequel $\frac{G_{h}^{0}(h)}{2}$ instead of $G_{h}^{0}(h)$. Thus, from (30), (31), (34) and (35):

$$
G_{h}(h)=\frac{1}{2 \frac{f_{2}^{h}}{f_{1}^{h}}+1}=\left[\frac{1}{6} \frac{R}{b} \frac{1+\frac{3 b}{R}}{1+\frac{2 b}{R}}\left[\left(\frac{h}{6 R} \frac{R}{b}+1\right) \ln \left(1+\frac{6 b}{R} \frac{R}{h}\right)-1\right]+1\right]^{-1}
$$

$G_{h}(h)$ appears as a function of $\frac{h}{R}$ parameterised by $\frac{b}{R}$.

Lastly, to calculate the collision efficiency coefficient $\alpha$ of two spherical grains in case of non-wetting, we have to put expression (36) into relation (32). This gives:

$$
\alpha_{h 1,2}=\left[24 \int_{2}^{\infty} \frac{e^{U}}{\boldsymbol{r}^{\prime^{4}} G\left(r^{\prime}\right)} d r^{\prime}\right]^{-1}
$$

with :

$$
U=18 \pi C_{A} \frac{1+\frac{3 b}{a}}{1+\frac{2 b}{a}} \int_{\infty}^{r} \frac{1}{s^{2}} \frac{d V^{\prime}(s)}{d s} d s
$$

Figure 5 shows the variation of $\alpha^{h}{ }_{1,2}$ versus $C_{\mathrm{A}}$ for different values of $\frac{b}{R}$ 
Remark: in the previous reasoning, non-wetting is taken into account only via the new expression of $G(h)$; rigorously we should have replaced the Wan der Waals potential by the expression obtained from the Ruckenstein-Bjerkness approach (13). This would have led us to heavy calculation that we can avoid by keeping the van der Waals expression and the derived parameter $C_{\mathrm{A}}(33)$, however by letting $C_{\mathrm{A}}$ vary over a large range of values. This point will be examined more quantitatively just below.

\section{iv) collision efficiency for aggregation of alumina in liquid steel}

Before estimating $\alpha_{1,2}^{h}$ for the alumina- liquid steel system (particles of radius $5 \mu \mathrm{m}$ ), we have to determine parameters $C_{\mathrm{A}}$ in the most common conditions of aggregation. The numerical values of the problem characteristics are shown in Table 2.

In the considered domain of $C_{\mathrm{A}}$ values $\alpha^{h}{ }_{1,2}$ is moderately sensitive to $C_{\mathrm{A}}$ (Figure 5). For instance, for hundred or thousand times as large Hamaker constants, $\alpha$ is at the highest multiplied by 2 .

In their respective works, Parker et al. [12] and Vinogradova [40] proposed $b$ values ranging between 0.1 and $0.5 \mu \mathrm{m}$. For our reference system, no estimation of slip length is available. Likely presence of gas pockets in the porosity as discussed before rather suggests higher values of $b$ (consistent with gas-liquid interface) and $b / R$ values. From Figure 5 , it is clear than $\alpha_{1,2}^{h}$ is an increasing function of parameter $b$. Table 3 highlights typical results. It appears that non-wetting significantly increases the collision efficiency, thus of the aggregation rate in the same experimental conditions. Effect is more pronounced at lower turbulence intensity.

Remark: The interaction forces due to the presence of gas cavities (see above Ruckenstein model (13)) are certainly not of the same mathematical form as van der Waals forces, however, as their intensity is probably not much lower nor higher, the previous calculations (Figure 5; Table 3) are valid in this case too, at least to give a good approximation of 
$\alpha^{h}{ }_{1,2}$ values with a corrected Hamaker constant value in $C_{\mathrm{A}}$ that we obtain by multiplying original value of $C_{\mathrm{A}}$ by factor $\left(r_{\mathrm{B} / \mathrm{W}}+1\right)$.

\section{Fragmentation}

Fragmentation is often invoked to explain that a maximum aggregate size is almost always observed in the aggregation processes [7, 45]. The occurrence of break-up depends on the balance between the disaggregation effects due to the action of the fluid and the overall cohesion of the aggregate due to the interactions between primary particles.

In the present case of non-wetting, the situation of fragmentation is quite new because, as we saw before, the different particles are linked by gas bridges. To our knowledge this problem has not been studied yet.

In this paper, we only propose a preliminary discussion and examine the fragmentation conditions of a doublet of identical spherical particles in two stages of its formation, i.e., just after the collision between the particles and later when the gas bridge is built up.

\section{- particle "adhesion"}

In what follows, we assume that gas cavities pre-exist at or under the surface of the solid particles.

The building up of a gaseous bridge between two neighbouring inclusions requires that the two colliding particles stay close to each other for a time sufficient to form and spread this gaseous link on the contact surface. Similar situations have been envisaged for agglomeration from supersaturated solutions [46, 47] and for coalescence processes in liquid-liquid dispersions [48]. The methods used by the authors, in spite of their differences, consist in comparing the time $t_{\mathrm{b}}$ needed to form the bridge (whatever its nature) and the time $t_{\mathrm{p}}$ spent by the particles in close proximity. 
For particles and aggregates smaller than the Kolmogorov microscale and thus submitted to the shear rate $\dot{\gamma}, t_{\mathrm{p}}$ is currently taken equal to $\dot{\gamma}^{-1}$, thus:

$$
t_{\mathrm{p}} \propto\left(\frac{v}{\varepsilon}\right)^{1 / 2}
$$

To estimate $t_{\mathrm{b}}$, we consider a system of two inclusions plane surfaces separated by a narrow gap (Figure 4). This type of problem has already been tackled, however, at the molecular scale [49]. We propose here a macroscopic hydrodynamic approach. Time $t_{\mathrm{b}}$, the drainage time of the liquid film is also the propagation time of the toroidal liquid-gas meniscus in this configuration (which is assumed axisymmetric). The motion of the meniscus is due to the pressure difference $\Delta P$ between the gas-liquid interface and the liquid phase outside the slit, i.e., according to previous developments in this paper:

$$
\Delta P=\left(P_{\mathrm{sat}}+\gamma_{\mathrm{LG}} \frac{\cos \theta}{R_{c}}\right)-P_{\mathrm{ext}}
$$

$R_{\mathrm{c}}$ is the curvature radius of the meniscus. Let $H_{M}$ be the radius of each inclusion surface $\left(H_{M} \approx 2.5 \mu \mathrm{m}\right)$. Gas bubbles are assumed to be present in the vicinity of the surface in underlying cavities (as seen before) and can feed the slit with gas. To calculate the time needed for the gas pocket to develop from size zero to size $H_{\mathrm{M}}$, we have to solve the following equation system:

i) Navier-Stokes equation (radial component in cylindrical coordinates))

$$
\rho\left(\frac{\partial v_{r}}{\partial t}+v_{r} \frac{\partial v_{r}}{\partial r}\right)=-\frac{\partial P}{\partial r}+\mu\left(\frac{\partial}{\partial r}\left(\frac{1}{r} \frac{\partial\left(r v_{r}\right)}{\partial r}\right)+\frac{\partial^{2} v_{r}}{\partial z^{2}}\right)
$$

ii) flow-rate conservation

$$
r v_{\mathrm{r}}=f(t, z)
$$


$r$ is the radial coordinate, $z$, the transversal coordinate, $v_{\mathrm{r}}$ is the radial velocity (the only non-zero component of the velocity), $\mu$, the fluid dynamic velocity, $\rho$, its density and $f$ a function of only time $t$ and $z$.

If we assume too that the drainage flow is laminar (parabolic profile in $z$ ), previous system can be solved. We obtain in particular:

$$
t_{b}=\frac{3 \mu H_{M}^{2}}{4 \gamma_{L G}|\cos \theta| R_{c}}
$$

Numerical values of parameters in Equations (38) and (42) have been already specified except $R_{\mathrm{c}}$ which is difficult to estimate. For $R_{\mathrm{c}}=1 \mu \mathrm{m}, t_{\mathrm{b}}=1.9 \times 10^{-8} \mathrm{~s}$; for $R_{\mathrm{c}}=0.01 \mu \mathrm{m}$, $t_{\mathrm{b}}=1.9 \times 10^{-6} \mathrm{~s}$; this latter value is certainly the highest possible value of $t_{\mathrm{b}}$ as lower values of $R_{\mathrm{c}}$ can be hardly envisaged (because of probable surface asperities for instance). As, on the other hand, $t_{\mathrm{p}}$ ranges between $1.7 \times 10^{-3}$ and $5 \times 10^{-2} \mathrm{~s}$, it is quite clear that the time needed to build up a gas bridge is considerably shorter than the duration of the contact. Thus sticking probability of the two inclusions is close to 1 , once contact has occurred.

\section{- doublet rupture}

The authors generally express the competition between the disaggregation and the cohesion effects using the ratio $\frac{\sigma}{\tau} ; \sigma$ is the mean mechanical strength of the aggregate and $\tau$ is the mean shear [50-52]. The breakage rate is proportional to $\dot{\gamma} e^{-\frac{\sigma}{\tau}}$. The breakage rate depends on the hydrodynamic conditions of the flow, via the energy dissipation rate $\varepsilon$ and the dynamic viscosity $\mu$, and on the characteristics of the aggregates: outer radius, fractal dimension, primary particle radius and cohesion force between two primary particles.

The action of a shear flow on a doublet of spherical particles has been studied by several authors $[53,54]$. Another interesting bibliographic source concerns the studies of rupture 
of pendular (liquid) bridges between particles (Simmons et al., 1994) which present a certain similarity with the investigated problem. As previously, we do not present here a complete study of the fragmentation phenomenon by breakage of the bridge, but, we only submit qualitative aspects.

In Equation (18), we gave the expression of the force acting between two particles linked by a gas bridge. This force can be compared to the tensile force acting on a doublet in planar linear shear field [54].

$$
F_{\mathrm{D}} \approx 4 \lambda \dot{\gamma} \mu R^{2}
$$

where coefficient $\lambda$ is equal to 4.83 for particles in contact.

For common parameter values of the liquid steel-alumina system and $r_{1}=r_{2}=2 \mu \mathrm{m}$ and $R=2.5 \mu \mathrm{m}:$

$F_{\mathrm{G}}=2.1 \times 10^{-5} \mathrm{~N}$ and $F_{\mathrm{D}}=3 \times 10^{-10}$ to $9 \times 10^{-9} \mathrm{~N}$.

It is quite clear that, even if the approximations used here are simplistic, the disruption force of the fluid is considerably lower than the cohesion force of the doublet and that fragmentation of a gaseous bridge by the liquid flow seems to be very unlikely. The consequence is that very large aggregates can be expected in aggregation in non-wetting media.

\section{CONCLUSION}

In this paper we propose a set of theoretical considerations in the aim of building a model of aggregation of a solid in a non-wetting liquid. From thermodynamic considerations we prove that gas pockets can spontaneously form in the underlying cavities of the solid-liquid surface. These cavities play an essential part in the formation of gas bridges between 
particles in contact. The role of this interfacial gas layer is examined in three steps of the aggregation mechanism:

i) the possible hydrodynamic force which results from coupled pulsations between gas cavities is proved to be lower or larger than van der Waals force according to the problem parameters, however probably never high enough to noticeably modify the aggregation kinetics ;

ii) liquid drainage between approaching particles is facilitated and thus collision efficiency is increased (approximately multiplied by 2);

ii) particle adhesion via the formation of the gas bridge is very rapid and does not influence the aggregation dynamics;

iii) the presence of a gas bridge between solid particles gives to the agglomerates a very strong cohesion which considerably reduces fragmentation.

These new elements of interpretation have been introduced into the expressions of the aggregation and fragmentation kernels to give a comprehensive model of aggregation in non-wetting medium. Accurate validation of the previous theoretical approach on the investigated system alumina-liquid steel present obvious difficulties. Photographs, however, are available and confirm the presence of several hundreds of micrometers inclusion clusters. Integration of the dynamical part of our model in global codes of computational fluid dynamics (CFD) has been performed and gives good indications for the aggregates spatial distribution [19].

In a recent work, we presented an experimental study concerning aggregation of hydrophobic silica in water-ethanol mixtures and validated the present model in a correct way [23]. Aggregation was followed by turbidimetry. Figures 6 shows the best agreement which was found between experimental and calculated turbidity curves vs time in typical case of 
aggregation of $0.5 \mu \mathrm{m}$ hydrophobic silica at stirring rate $400 \mathrm{rpm}$. Slip length is equal to 0.05 $\mu \mathrm{m}$. Aggregates are relatively compact (fractal dimension 2.7) and contain a volume fraction in gas of 0.3. The procedure of determination of these two characteristics is explained in [23]. From a practical point of view, application and validation of the present model in the general experimental situation of a solid granular sample aggregating in non-wetting medium proceeds according to the following steps:

i) collection of the product and system characteristics, in particular: solid particle size distribution, Hamaker constant, turbulent energy dissipation rate;

ii) confirmation of the non-wetting conditions from contact angle measurement (when possible) or calculation according to equation (1);

iii) estimation of the ratio hydrophobic force/van der Waals force using equation (14) and possible modification of coefficient $C_{\mathrm{A}}$;

iv) determination of the aggregation kernel elements: collision frequency (20) and (21) after calculation of the collision efficiency coefficient (37);

v) determination of the fragmentation conditions: verification of the instantaneity of particle bridging (equations (38) and (42)) and validation of the zero fragmentation kernel (equations (18) and (43)).

The so calculated aggregation and fragmentation kernels should be then put in the population balance equation [55] from which the particle size distribution variation vs. time can be calculated.

Lastly, from comparison with the available experimental results, model validation and identification of possible unknown parameters can be obtained. 


\section{NOTATION}

\begin{tabular}{|c|c|}
\hline$A$ & Hamaker constant \\
\hline$b$ & slip length \\
\hline$C_{\mathrm{A}}$ & dimensionless number (Eqn [33]) \\
\hline$d$ & depth penetration (Eqn (9)) \\
\hline$D_{\mathrm{b}}$ & Brownian diffusivity \\
\hline$f_{1}$ & friction coefficient \\
\hline$f_{2}$ & friction factor \\
\hline$F$ & force \\
\hline$F_{\mathrm{D}}$ & tensile force acting on a doublet in planar linear shear field \\
\hline$F_{\mathrm{G}}$ & force acting between two spheres linked by a gas bridge \\
\hline$F_{\mathrm{SS}}$ & hydrodynamic force between two spheres \\
\hline$F_{\mathrm{G}}$ & van der Waals force \\
\hline$F_{1}$ & capillary force \\
\hline$F_{2}$ & pressure force \\
\hline$G, G^{0}$ & interaction correction coefficient \\
\hline$\Delta G_{\mathrm{n}}$ & Gibbs free enthalpy variation \\
\hline$h$ & particle separation \\
\hline$h_{d}$ & dry length \\
\hline$H_{M}$ & inclusion radius \\
\hline$i, j$ & subscripts of granulometric classes \\
\hline$I$ & dimensionless integral \\
\hline$J$ & collision frequency \\
\hline$L$ & transversal width \\
\hline$n$ & mole number \\
\hline$n_{b}$ & number of gas cavity per unit surface area \\
\hline
\end{tabular}




\begin{tabular}{|c|c|}
\hline$P$ & function (defined in Eqn (5)) \\
\hline$P_{\text {ext }}$ & ambient pressure \\
\hline$P_{\mathrm{G}}$ & gas phase pressure \\
\hline$P_{\text {sat }}$ & saturation pressure \\
\hline$\Delta P$ & pressure deficiency \\
\hline$r$ & spherical coordinate \\
\hline$r_{\mathrm{B}}$ & bubble radius \\
\hline$r_{\mathrm{B} / \mathrm{W}}$ & force ratio \\
\hline$r_{1}, r_{2}$ & radii of curvature shown in Fig. 4 \\
\hline$R$ & particle radius \\
\hline$R_{C}$ & meniscus curvature radius \\
\hline $\boldsymbol{R}$ & gas constant \\
\hline$\Delta S$ & surface area variation \\
\hline$t_{P}, t_{b}$ & characteristic time \\
\hline$T$ & temperature \\
\hline$V$ & interaction potential \\
\hline$V^{\prime}$ & dimensionless interaction potential \\
\hline$V_{\mathrm{G}}$ & volume of gas cavity \\
\hline$V, v$ & fluid velocity \\
\hline
\end{tabular}

Greek letters

$\begin{array}{ll}\alpha & \text { half-angle of a crevice } \\ \alpha_{\mathrm{ij}}, \alpha_{12} & \text { collision efficiency coefficient } \\ \alpha^{h}{ }_{1,2} & \text { collision efficiency coefficient (in case of non-wetting) } \\ \beta & \text { proportionality coefficient } \\ \gamma & \text { interfacial tension }\end{array}$




$\begin{array}{ll}\dot{\gamma} & \text { gradient velocity } \\ \delta & \text { amplitude of the volume oscillations of the bubbles } \\ \varepsilon & \text { turbulent energy dissipation rate } \\ \varepsilon \mathrm{p}, \varepsilon & \text { proportionality constants } \\ \theta & \text { contact angle } \\ \mu & \text { liquid dynamic viscosity } \\ v & \text { kinematic viscosity } \\ \xi & \text { dimensionless constant (Eqn (15)) } \\ \rho & \text { liquid density } \\ \sigma & \text { mechanical strength of the aggregate } \\ \tau & \text { shear stress } \\ \omega & \text { oscillation phase difference } \\ & \text { bubble oscillation pulsation }\end{array}$




\section{REFERENCES}

[1] M. Z. Smoluchowski, Drei vorträge über diffusion, brownsche molekularbewegung und koagulation von kolloidteilchen, Z. Physik.Chem., 17(1916) 585-599.

[2] H. R. Kruyt, Colloid Science, Vol I, , Elsevier, Amsterdam, 1952, p 257.

[3] P. G. Saffman, J. S. Turner, On the collision of drops in turbulent clouds, J. Fluid Mech., 1(1956)16-30.

[4] L. A. Spielman, Viscous interactions in brownian coagulation, J. Colloid Interface Sci., 33(1970)562.

[5] T. G. Van de Ven, S. G. Mason, The microrheology of colloidal dispersions. VII. Orthokinetic doublet formation of spheres, Colloid and polymer Sci., 255 (1977) 468-479.

[6] K. Higashitani, R. Ogawa, G. Hosokawa, Y. Matsuno, Kinetic theory of shear coagulation for particles in a viscous fluid, J. Chem. Engng. Japan, 15(1982) 299-304.

[7] G.B.J. De Boer, G.F.M. Hoedemakers, D. Thoenes, Coagulation in turbulent flow, Chem. Engng. Res. Des., 67(1989) 301-315.

[8] K.A. Kusters, J.G.Wijers, D.Thoenes, Aggregation kinetics of small particles in agitated vessels, Chem. Eng. Sci., 52 (1997)107-121.

[9] H. Saint-Raymond, F. Gruy, M. Cournil, Turbulent aggregation of alumina in water and n-heptane. J. Colloid Interface Sci., 202 (1998) 238-250.

[10] V.V. Yaminsky, V.S. Yushchenko, E.A. Amelina, E.D. Shchukin, E.D., Interaction between particles in a nonwetting liquid, J. Colloid Interface Sci., 96 (1983) 307-314.

[11] P.M. Claesson, H.K. Christenson., Very long range attractive forces between uncharged hydrocarbon and fluorocarbon surfaces in water, J. Phys. Chem., 92 (1988) 1650-1655.

[12] J.L. Parker, P.M. Claesson, P. Attard, Bubbles, cavities, and the long-ranged attraction between hydrophobic surfaces, J. Phys. Chem., 98 (1994) 8468-8480.

[13] B.W. Ninham, K. Kurihara, O.I. Vinogradova, Hydrophobicity, specific ion adsorption and reactivity, Colloids Surf. A, 123-124 (1997) 7-12.

[14] O.I. Vinogradova, N.F. Bunkin, N.V. Churaev, O.A.Kiseleva, A.V. Lobeyev, B.W. Ninham, Submicrocavity structure of water between hydrophobic and hydrophilic walls as revealed by optical cavitation, J. Colloid Interface Sci. 173 (1995) 443-447. 
[15] A. Carambassis, L.C. Jonker, P. Attard, M.W. Rutland, Forces measured between hydrophobic surfaces due to a sub-microscopic bridging bubble, Phys. Rev. Lett., 80 (1998) 5357-5360

[16] G.E Yabukov, H.J.Butt, O.I. Vinogradova, Interaction forces between hydrophobic surfaces. Attractive jump as an indication of formation of stable microcavities, J. Phys. Chem. B, 104 (2000) 3407-3410.

[17] P. Attard, Thermodynamic analysis of bridging bubbles and a quantitative comparison with the masured hdrophobic atraction, Langmuir, 16 (2000) 4455-4466.

[18] P. Attard, Nanobubbles and the hydrophobic attraction, Adv. In Coll. And Inter. Sci., 104 (2003) 75-91.

[19] M. Cournil, F. Gruy, P. Gardin, H. Saint-Raymond, Experimental study and modeling of inclusion aggregation in turbulent flow to improve steel cleanliness, Phys. Stat. Sol. (a), 189 (2002) 159-168.

[20] P. Kozakévitch, L.D. Lucas, Rôle des phénomènes de surface dans l'élimination d'inclusions solides d'un bain métallique, Revue de Métallurgie, 65 (1968) 589-598.

[21] P. Kozakévitch, M. Olette, Rôle des phénomènes superficiels dans le mécanisme d'élimination des inclusions solides, Revue de Métallurgie, 68 (1971) 635-646.

[22] S.T. Johansen, S. Taniguchi, Prediction of agglomeration and break-up of inclusions during metal refining in Barry Welch (ed), Light Metals, TMS, 1998, 855-861.

[23] F. Gruy, M. Cournil, P. Cugniet, Influence of nonwetting on the aggregation dynamics of micronic solid particles in a turbulent medium, J. Colloid Interface Sci.(2005) in press.

[24] R. Defay, I. Prigogine, Tension superficielle et adsorption, Desoer, Liège, 1951.

[25] V.V. Yaminsky, B.W. Ninham, Hydrophobic force: lateral enhancement of subcritical fluctuations. Langmuir, 9 (1993) 3618-3624.

[26] E. Ruckenstein, N. Churaev, A possible hydrodynamic origin of the forces of hydrophobic attraction, J. Colloid Interface Sci., 147(1991), 535-538.

[27] B.V. Derjaguin, L. Landau, Acta Physiochim. URSS 14 (1941) 633. 
[28] E.J.W. Verwey, J.T.G. Overbeek, Theory of the Stability of Lyophobics Colloids, Elsevier, Amsterdam, 1948.

[29] H. C. Hamaker, Physica, 4 (1937) 1058.

[30] E. W. Lifshitz, Soviet Phys., JETP 2 (1956) 73.

[31] J.L. Parker, P.M. Claesson, Forces between hydrophobic silanated glass surfaces, Langmuir, 10 (1994) 635-639.

[32] J. Forsman, J., C.E. Woodward, B. Jönsson, Computer simulations of water between hydrophobic surfaces : the hydrophobic force, J. Phys. Chem., 100 (1996)1500515010.

[33] J. Forsman, J., C.E. Woodward, B. Jönsson, Repulsive hydration forces and attractive hydrophobic forces in a unified picture, J. Colloid Interface Sci., 195 (1997) 264266.

[34] E. Ruckenstein, The instability of the solid-liquid interface and the hydrophobic force. J. Colloid Interface Sci., 188 (1997) 218-223.

[35] L.D. Landau, E.M. Lifshitz, Fluid mechanics, Butterworth-Heinemann, Oxford, 1987.

[36] K. Hotta, K.Takeda, K. Iinoya, K., The capillary binding force of a liquid bridge, Powder Technology, 10 (1974) 231-242.

[37] G. Lian, C. Thornton, M.J. Adams, M. J., A theoretical study of the liquid bridge forces between two rigid spherical bodies, J. Colloid Interface Sci., 161 (1993)138-147.

[38] S. J. R. Simmons, J. P. K. Seville, M. J. Adams, An analysis of the rupture energy of pendular liquid bridges, Chem. Eng. Sci., 49 (1994) 2331-2339.

[39] E. Ruckenstein, P. Rajora, On the no-slip boundary condition of hydrodynamics, $J$. Colloid Interface Sci., 96 (1983) 488-491.

[40] O.I. Vinogradova, Coagulation of hydrophobic and hydrophilic solids under dynamic conditions, J. Colloid Interface Sci., 169 (1995) 306-312.

[41] O.I. Vinogradova, Slippage of water over hydrophobic surface, Int. J. Miner. Process, 56 (1999) 31-60.

[42] R.B. Bird, W.E. Stewart, E.N. Lightfoot, Transport phenomena, Wiley, New-York, 1960. 
[43] B.V. Derjaguin, V.M. Muller, Slow coagulation of hydrophobic colloids, Doklady Akademic Nauk SSSR, 176 (1967) 869-872.

[44] H. Saint-Raymond., F. Gruy, Turbulent coagulation efficiency, J. Colloid Interface Sci., 185 (1997) 281-284.

[45] V. Oles, Shear-induced aggregation and breakup of polystyrene latex particles, $J$. Coll. Interface Sci., 154 (1992) 351-358.

[46] R. David, P. Marchal, J.P. Klein, J.Villermaux, J., Crystallization and precipitation engineering - A discrete formulation of the agglomeration rate of crystals in a crystallization process, Chem. Eng. Sci., 46(1991) 205-213.

[47] H.S. Mumtaz, M.J. Hounslow, N.A. Seaton, W.R. Paterson, Orthokinetic aggregation during precipitation: a computional model for calcium oxalate monohydrate. Trans. Instn Chem. Engng, 75, Part A (1997) 152-159.

[48] A.K. Chesters, The modelling of coalescence processes in fluid-liquid dispersions: a review of current understanding, Trans. Instn Chem. Engng, 69, Part A (1991)259-270.

[49] X. Huang, C.J. Margulis, J. Berne, Dewetting-induced collapse of hydrophobic particles, PNAS, 100 (2003) 11953-11958.

[50] R.C. Sonntag, W.B. Russel, Structure and breakup of flocs subjected to fluid stresses. II. Theory, J. Colloid Interface Sci., 115 (1987) 378-389.

[51] P. Ayazi Shamlou, S. Stavrinides, N. Titchener-Hooker, M. Hoare, Growthindependent breakage frequency of protein precipitates in turbulently agitated bioreactors, Chem. Eng. Sci., 49 (1994) 2647.

[52] H. Luo, H.F. Svendsen, H. F., Theoritical model for drop and bubble breakup in turbulent dispersions. AIChE J., 42 (1996) 1225-1233.

[53] S. Wakiya, Slow motion in shear flow of a doublet of two spheres in contact, Journal of the physical society of Japan, 31 (1971) 1581-1587.

[54] S.P. Tha, H.L. Goldsmith, Interaction forces between red cells agglutinated by antibody, Biophys. J., 50 (1986) 1109-1116.

[55] Randolph, A. D., and Larson, M. A., “ Theory of Particulate Processes ”, Academic Press, NewYork (1988). 


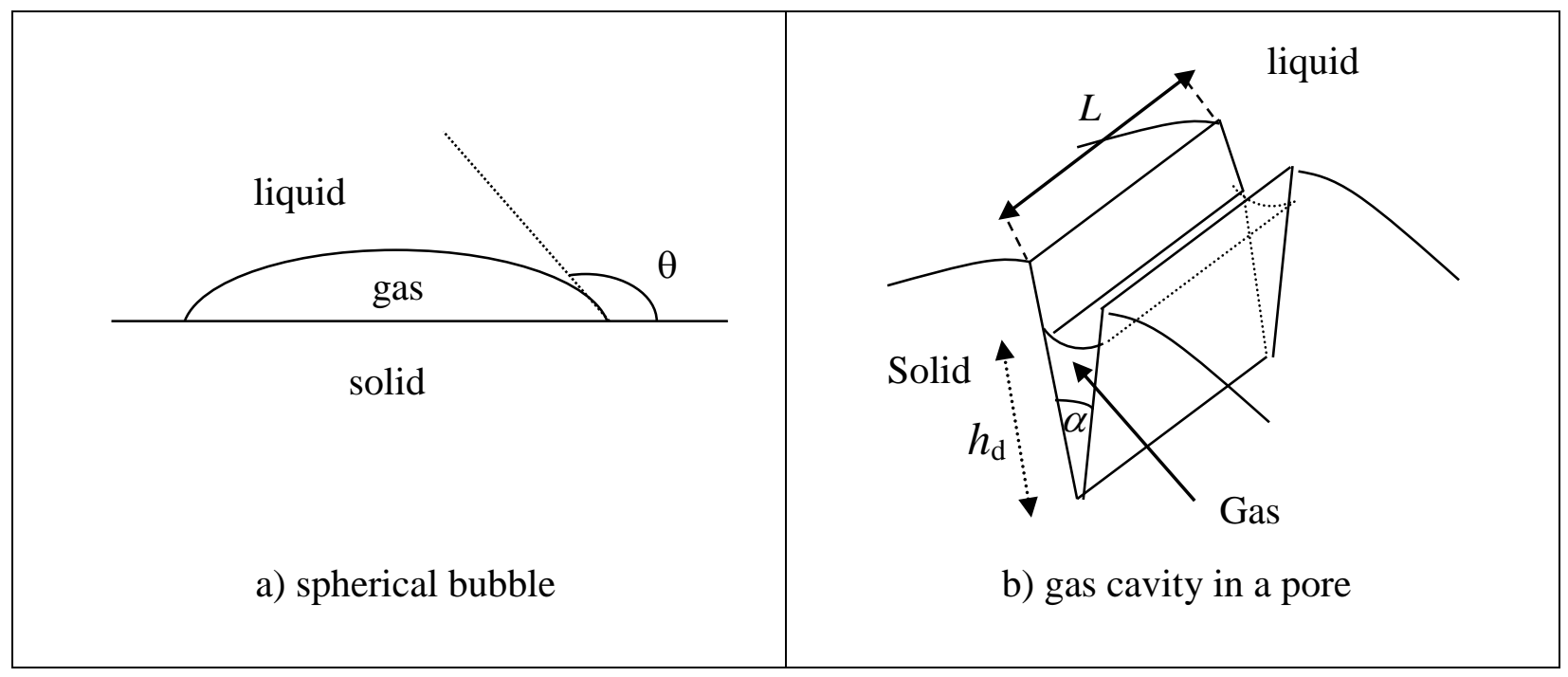

Figure 1: Different 3-phase configurations 
Liquid phase

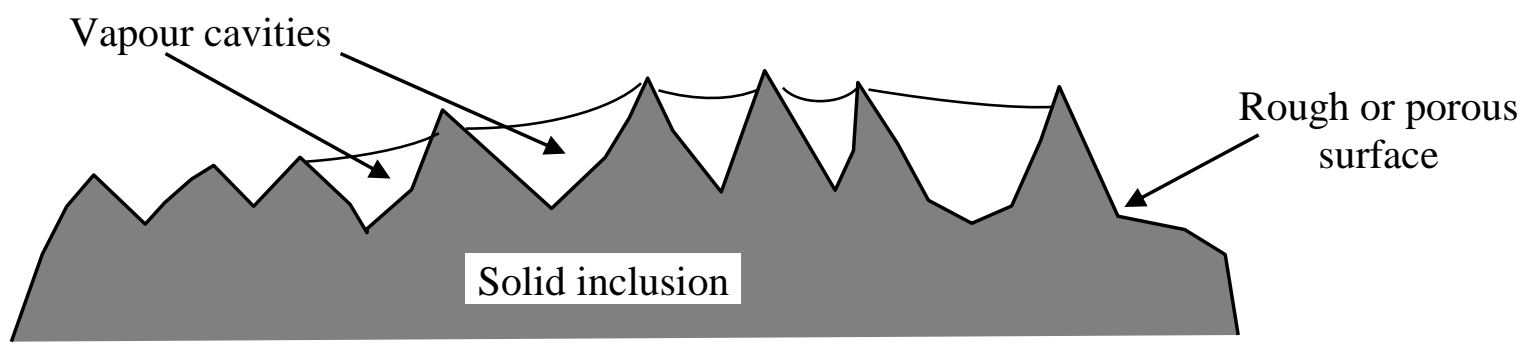

Figure 2: Representation of the solid-liquid interface with gas cavities

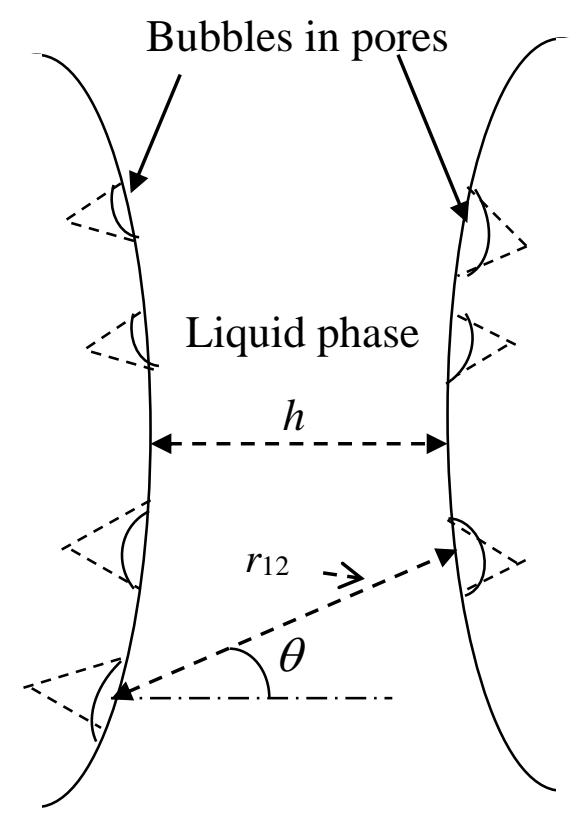

Figure 3: Geometrical position of two plane surfaces in interaction 


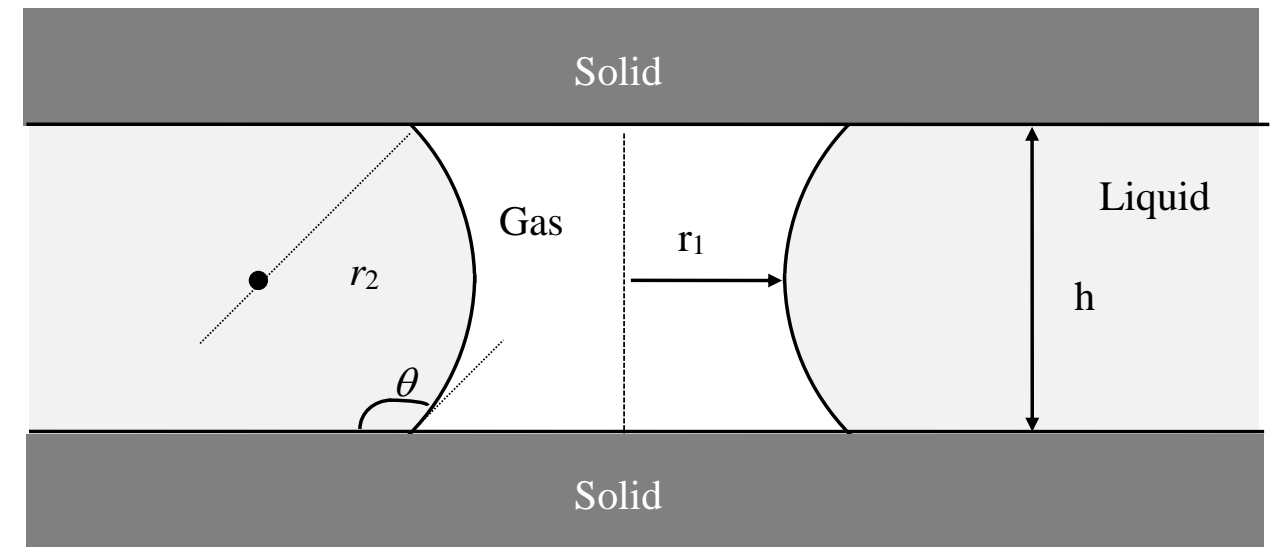

Figure 4: Toroidal gas bridge between two flat interfaces 


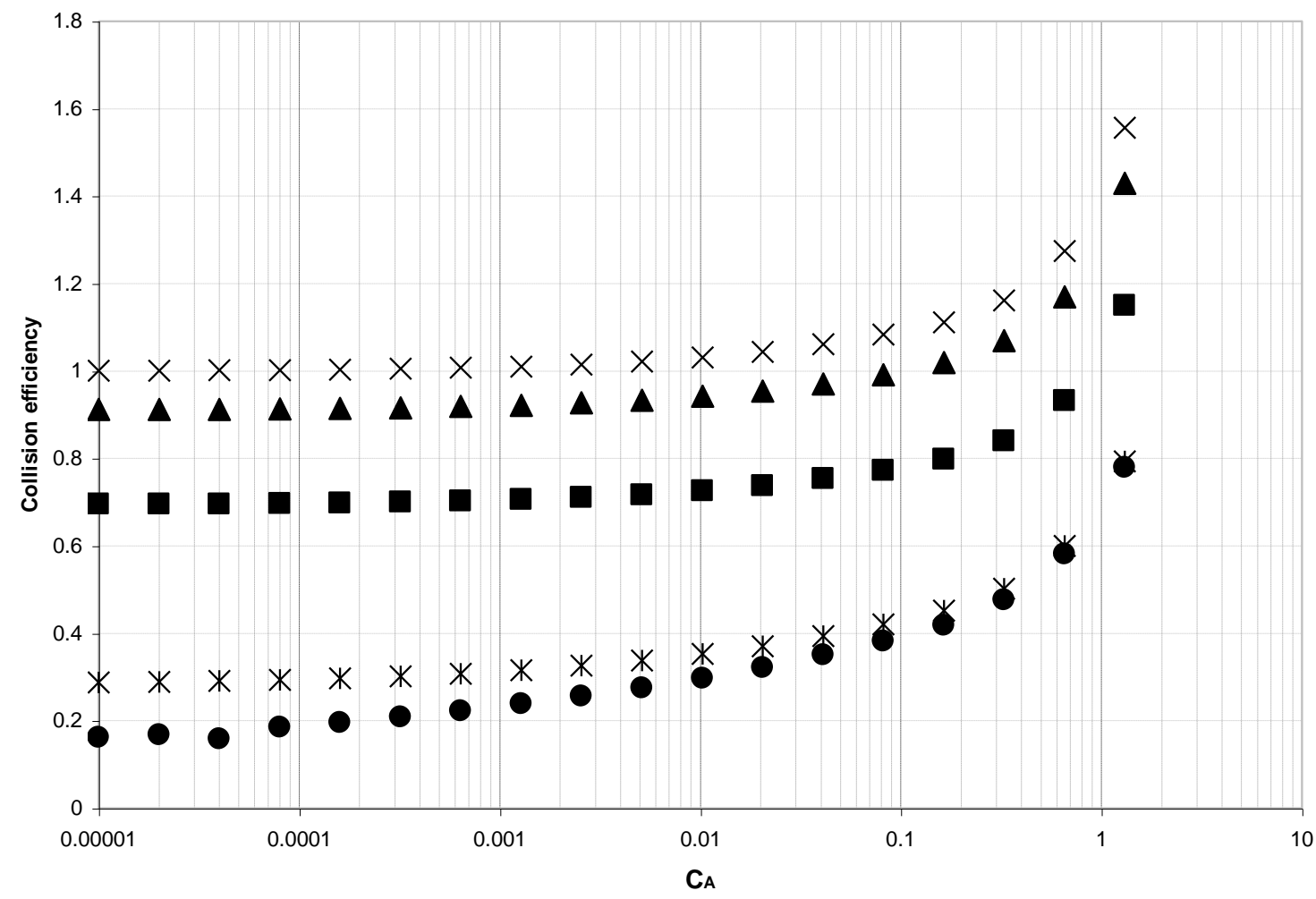

$x b / R=100000$ $\Delta \mathrm{b} / \mathrm{R}=10$

- $\mathrm{b} / \mathrm{R}=1$

$* \mathrm{~b} / \mathrm{R}=10-2$

- $b / R=10-4$

Figure 5: Variation of collision efficiency $\alpha$ versus $C_{\mathrm{A}}$ for different values of $\frac{b}{R}$ 


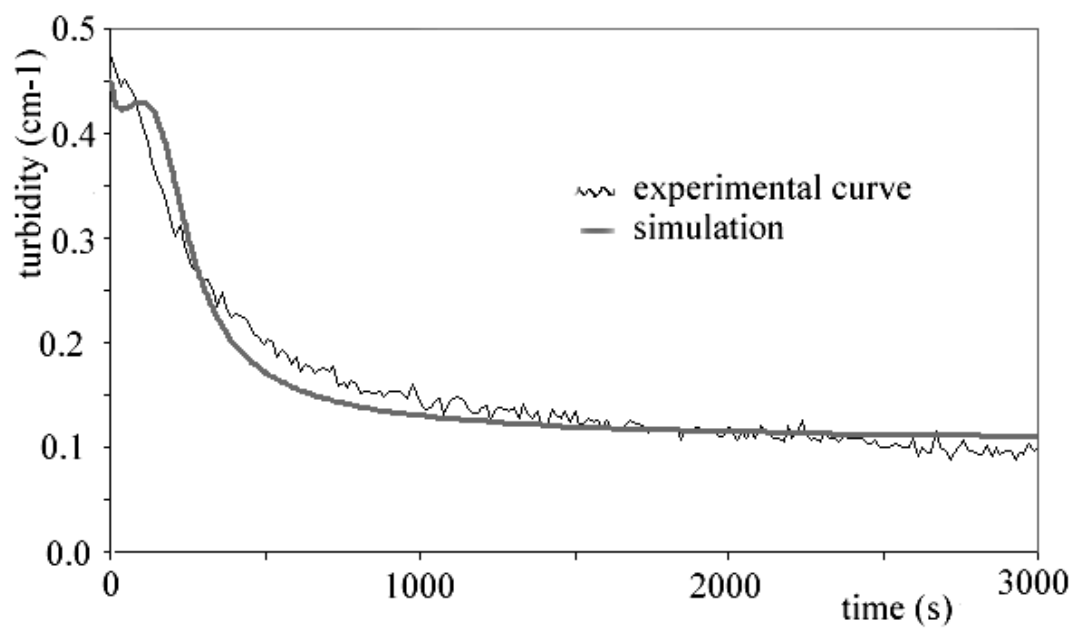

Figure 6: Aggregation of $0.5 \mu \mathrm{m}$ hydrophobic silica at $400 \mathrm{rpm}$ : experimental and simulated turbidity curves 


\begin{tabular}{|c|c|c|c|c|c|c|c|}
\hline$d(\mu \mathrm{m})$ & 0.001 & 0.003 & 0.01 & 0.03 & 0.1 & 0.3 & 1 \\
\hline$F_{\mathrm{B}} / F_{\mathrm{w}}$ & $1.56 \times 10^{-4}$ & $2.6 \times 10^{-3}$ & 0.1 & 3.2 & 140 & $4.7 \times 10^{3}$ & $2.7 \times 10^{5}$ \\
\hline
\end{tabular}

Table 1: Maximum value of $\mathrm{F}_{\mathrm{B}} / \mathrm{F}_{\mathrm{w}}$ for different $d$ values ; $R=1 \mu \mathrm{m}$

\begin{tabular}{|c|c|}
\hline Energy dissipation rate $\varepsilon$ & $10^{-3}-1 \mathrm{~m}^{2} \cdot \mathrm{s}^{-3}$ \\
\hline Kinematic viscosity $v$ & $0,7 \times 10^{-6} \mathrm{~m}^{2} \cdot \mathrm{s}^{-1}$ \\
\hline Density & $7 \times 10^{3} \mathrm{~kg} \cdot \mathrm{m}^{-3}$ \\
\hline Velocity gradient $\dot{\gamma}$ & $18.9-598 \mathrm{~s}^{-1} \cdot$ \\
\hline Hamaker constant $A$ & $1.0 \times 10^{-19} \mathrm{~J}$ \\
\hline Constant $C_{\mathrm{A}}$ & $3 \times 10^{-4}-1.0 \times 10^{-2}$ \\
\hline
\end{tabular}

Table 2: Numerical values of different physical characteristics of the aggregation problem

\begin{tabular}{|c|c|c|}
\hline $\begin{array}{c}b / R=1 \\
\text { (non-wetting) }\end{array}$ & $C_{\mathrm{A}}=3 \times 10^{-4}$ & $\alpha \approx 0.70$ \\
\cline { 2 - 3 } & $C_{\mathrm{A}}=1.0 \times 10^{-2}$ & $\alpha \approx 0.73$ \\
\hline $\begin{array}{c}b / R=0.1 \\
\text { (non-wetting) }\end{array}$ & $C_{\mathrm{A}}=3 \times 10^{-4}$ & $\alpha \approx 0.50$ \\
\cline { 2 - 3 } & $C_{\mathrm{A}}=1.0 \times 10^{-2}$ & $\alpha \approx 0.58$ \\
\hline \multirow{2}{b}{$\begin{array}{c}\text { (wetting) } \\
\text { (wo }\end{array}$} & $C_{\mathrm{A}}=3 \times 10^{-4}$ & $\alpha \approx 0.15$ \\
\cline { 2 - 3 } & $C_{\mathrm{A}}=1.0 \times 10^{-2}$ & $\alpha \approx 0.25$ \\
\hline
\end{tabular}

Table 3: Collision efficiency $\alpha$ for wetting and non-wetting and different particle size $(R=5$ $\mu \mathrm{m})$ 